\title{
Retrieval of foreign body bronchus in paediatric age group: a challenge
}

\author{
Gunjan Dwivedi ${ }^{1}$, Vikas Gupta ${ }^{1}$, Deepak Dwivedi ${ }^{2}$, Amit Sood $^{1}$, \\ Madhukar Upadhyay ${ }^{1}$, Manoj Kumar ${ }^{1}$
}

\begin{abstract}
${ }^{1}$ Department of ENT-HNS, ${ }^{2}$ Department of Anesthesia, Critical care and Pain management, Command Hospital, Armed Forces Medical College, Pune, Maharashtra, India
\end{abstract}

Received: 22 May 2018

Accepted: 12 June 2018

\author{
*Correspondence: \\ Dr. Vikas Gupta, \\ E-mail:vg.enthns@gmail.com
}

Copyright: () the author(s), publisher and licensee Medip Academy. This is an open-access article distributed under the terms of the Creative Commons Attribution Non-Commercial License, which permits unrestricted non-commercial use, distribution, and reproduction in any medium, provided the original work is properly cited.

\begin{abstract}
Foreign body (FB) aspiration can be a cause of serious morbidity and occasional mortality in pediatric population. Sometimes the diagnosis is delayed when the history of FB aspiration is not forthcoming, not witnessed or when parents fail to appreciate the symptoms. Unless there is a high degree of suspicion these cases may easily be missed and may be treated for a prolonged duration as upper respiratory tract infection (URTI) or pneumonia. A careful history plus a high degree of clinical suspicion is crucial. Involvement of other specialties is the key to diagnose and remove a FB from airway successfully. We present a case series of three cases of FB bronchus and challenges faced during their retrieval. The aim of this report is to highlight the importance of history taking, meticulous preparation, and precision required to remove a foreign body bronchus..
\end{abstract}

Keywords: Foreign body bronchus, Optical forceps, Rigid bronchoscopy, Virtual bronchoscopy, Paediatric anaesthesia

\section{INTRODUCTION}

Foreign body (FB) aspiration is a common cause of hospitalization in healthy children especially in children aged 1 to 3 years. ${ }^{1}$ Although most of the FB aspirations go unnoticed and do not pose an imminent threat to life, in certain cases, contrary can occur, causing a life threatening situation. The presentation depends on the type of object and degree of airway blockage. Most common presentation is sudden onset coughing and choking. Sometimes FB may have been lodged in the airway for a long time before first medical attention is sought. Serious complications occur if a FB remains in situ for a very long time. Commonly aspirated foreign bodies include organic materials like peanut, whereas older children have tendency to swallow metallic foreign bodies, however, the list is endless.

Rigid bronchoscopy is the standard technique for the removal of airway foreign body. Since the airway is shared by the ENT surgeon and the anesthesiologist during bronchoscopy, a good communication between the two is of paramount importance so as to chalk out an exhaustive procedural plan, leaving no space for untoward anxious moments to occur. As the airway in children is of very small caliber, it warrants some degree of skill and composure to negotiate rigid bronchoscope and procedure per se. Availability of optical forceps which can be guided through rigid ventilating bronchoscope immensely aid in simplifying the procedure as well as makes the procedure less hazardous.

We report a case series of FB bronchus with special emphasis on the anaesthetic management and meticulous removal of the foreign body.

\section{CASE PRESENTATION}

\section{Case 1}

A, two year old child presented to paediatric OPD with history of cough for 20 days and one episode of fever 03 
days back. The child had not been evaluated before the fever episode at any centre and did not have any respiratory distress. There was no history forthcoming of any foreign body ingestion, choking episode and cyanosis. The child had attained all milestones as per the age.

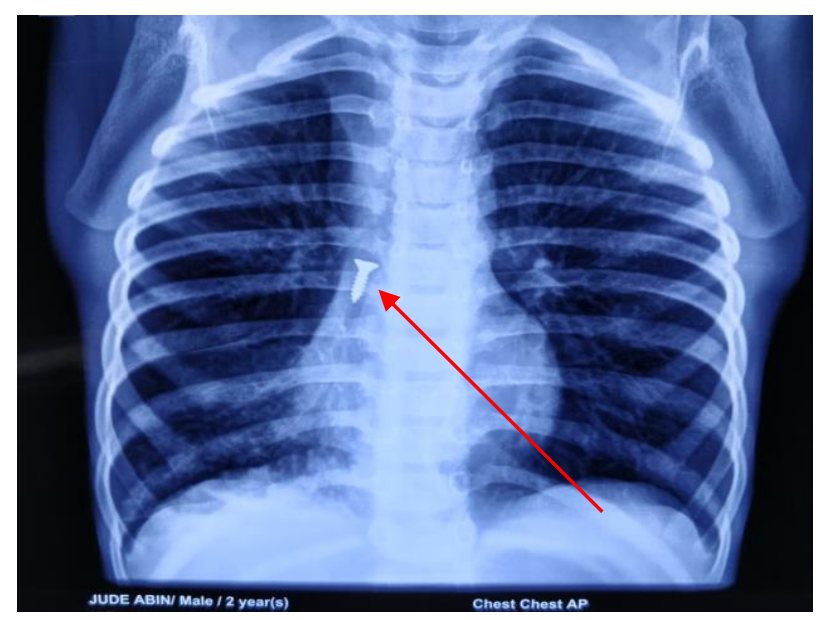

Figure 1: Metallic foreign body in Rt main bronchus.

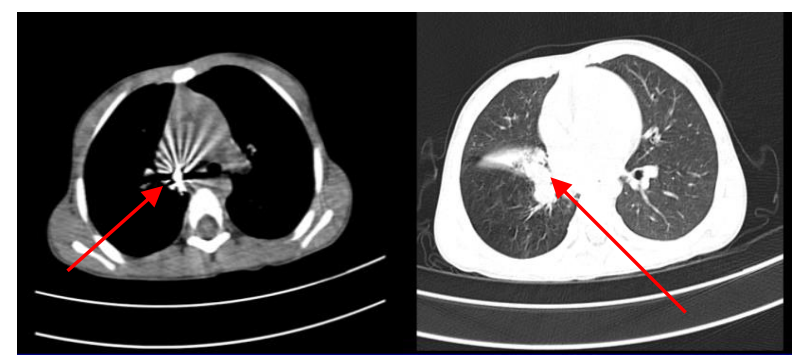

Figure 2: Foreign body with artifact and collapsed region of lung distal to $\mathrm{FB}$.

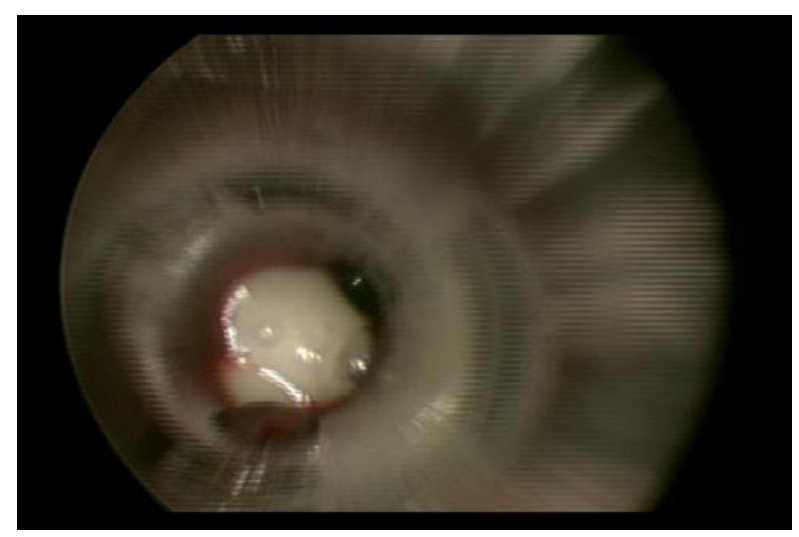

Figure 3: Foreign body covered with mucus plug.

On evaluation by paediatrician, the child was referred to our department, with chest x-ray showing FB in right main bronchus (Figure 1). There was slightly decreased air entry on the right side, however, there was no lung collapse on chest $\mathrm{x}$-ray. Since, status of lungs was not convincing, so a non-contrast computed tomogram
(NCCT) chest was done to look for any evidence of lung collapse as a result of bronchial obstruction and to pin point the location of foreign body. Owing to the metallic nature of the foreign body there were a lot of artifacts on the CT images. Foreign body was localized in right intermediate bronchus causing small area of collapse distal to the FB (Figure 2). An informed consent from the parents of the child was obtained for rigid bronchoscopy and removal of the foreign body.

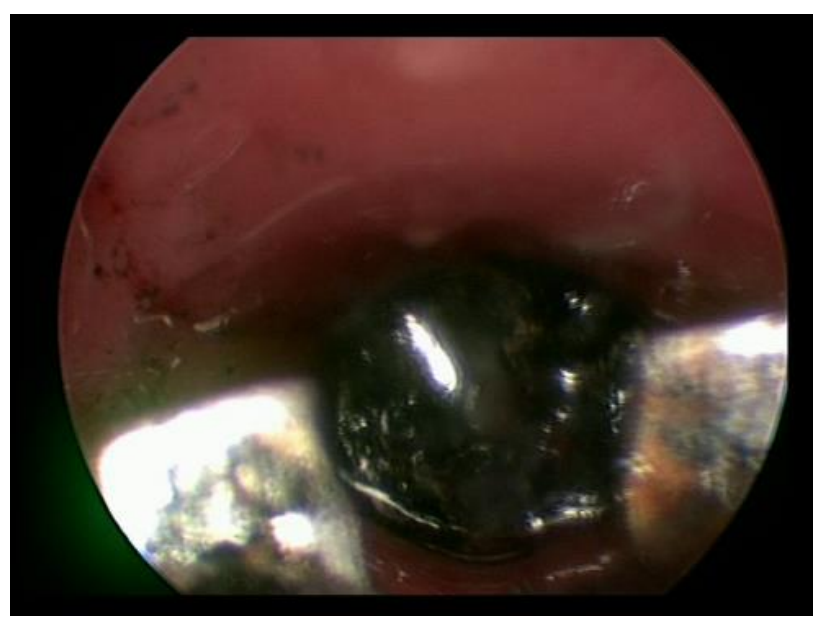

Figure 4: Screw completely blocking the subglottic airway.

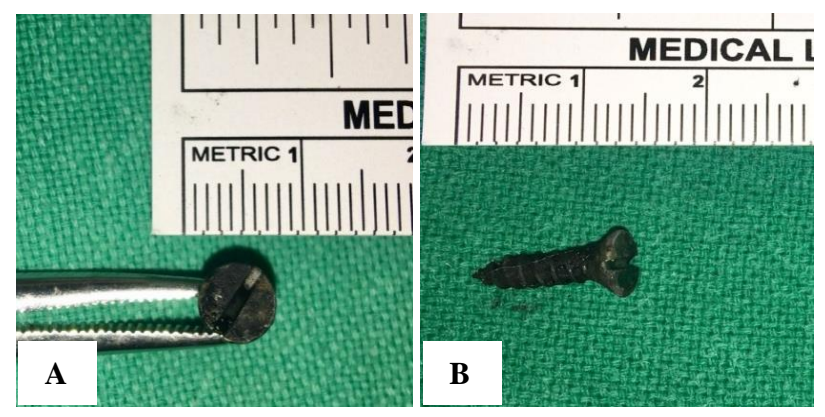

Figure 5: (A and B) Screw after removal: dimension of head and body.

After premedication with injection glycopyrrolate, anaesthesia was induced with injection propofol, and maintained with oxygen, air and sevoflurane. Optimal muscle relaxation was achieved with injection succinylcholine. $4.5 \mathrm{~mm}$ ID rigid bronchoscope (Karl Storz) was negotiated successfully beyond the vocal cords. Optical forceps was then introduced to localize the FB which was identified in right intermediate bronchus covered in mucus (Figure 3). After clearing the mucoid discharge, the FB, a metal screw, was held from edge of its head, with optical forceps and gently pulled upwards. However, at the level of subglottis the surgeon realized that the FB is held in a transverse position with the long axis of the FB perpendicular to the long axis of glottis and subglottic airway. At this moment the surgeon had to deliberately leave the FB, due to apprehension of causing injury to the subglottic lumen. With gentle manipulation 
the surgeon was able to align the axes of both the FB and the glottis in one direction and again tried to pull the FB upwards. However, this time the circular head of the foreign body, got stuck at the subglottis and accidently slipped off the grasp of optical forceps, completely obstructing the tracheal airway (Figure 4). The child started to de-saturate with $\mathrm{SpO} 2$ as low as $70 \%$. The surgeon pushed the screw downwards into the right main bronchus so that ventilation could be done through other bronchus. Retrieval was attempted again and the screw was held from its head. This time, the surgeon was able to pull out the screw and forceps as a single assembly out of the airway. A black colored metallic screw with head diameter of $6 \mathrm{~mm}$ and length of $1.1 \mathrm{~cm}$ was removed (Figure 5). It is important to note is that, subglottic dimension in a 2-3 year old child is around 5.5 to $6 \mathrm{~mm}$.

During the procedure child was ventilated through the ventilating rigid bronchoscope maintaining adequate depth of anaesthesia. Check bronchoscopy revealed few areas of linear abrasion on the tracheal wall and no evidence of any other FB in the airway. Post procedure child was intubated and vigorous physiotherapy was done on table to dislodge stagnant mucous. Later child was extubated and kept in paediatric intensive care unit (PICU), where he was managed with parentral antibiotics, nebulization with diluted adrenaline and budesonide along with chest physiotherapy.

\section{Case 2}

An 11 months old infant presented to the paediatric OPD with history of fever and cough of four days duration and high degree of suspicion of foreign body ingestion as per parents. There was no respiratory distress. Auscultation revealed decreased breath sounds on the right side of chest. Chest X-ray showed hyperinflation of the right lung with mediastinal shift to left (Figure 6). Impression of a foreign body lodged in right main bronchus was made.

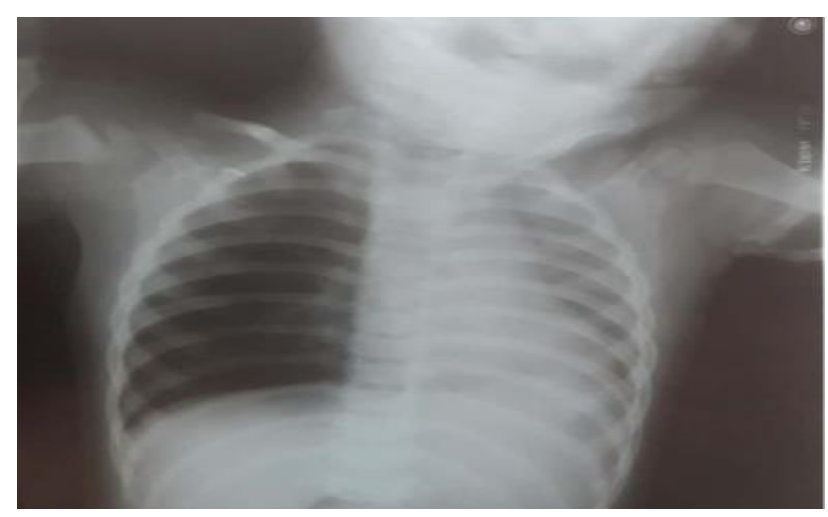

Figure 6: CXR showing hyperinflation of Rt lung with mediastinal shift to $\mathbf{L t}$.

Findings were further confirmed by an NCCT chest, which revealed a foreign body lodged in the right main bronchus probably of vegetative origin. The child was taken up for bronchoscopy and FB removal with optical forceps under general anaesthesia (GA) with similar technique as described in case 1. Foreign body was visualized which was embedded in mucosal lining surrounded with oedema and granulation tissue. Foreign body was grasped and removed in toto (Figure 7).

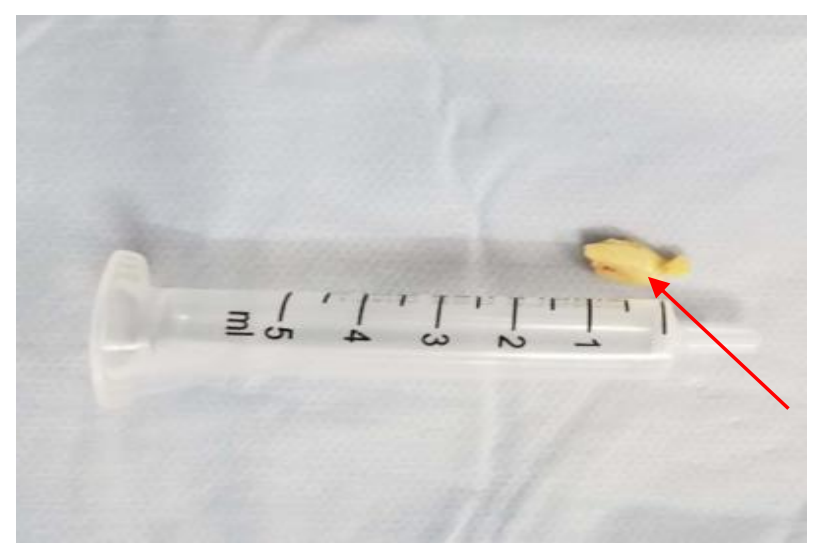

Figure 7: Foreign body (Ground nut).

Check bronchoscopy revealed no evidence of any residual FB and no bleeding anywhere in the airway. Post procedure child was comfortable and started on parentral antibiotics, frequent nebulization and chest physiotherapy.
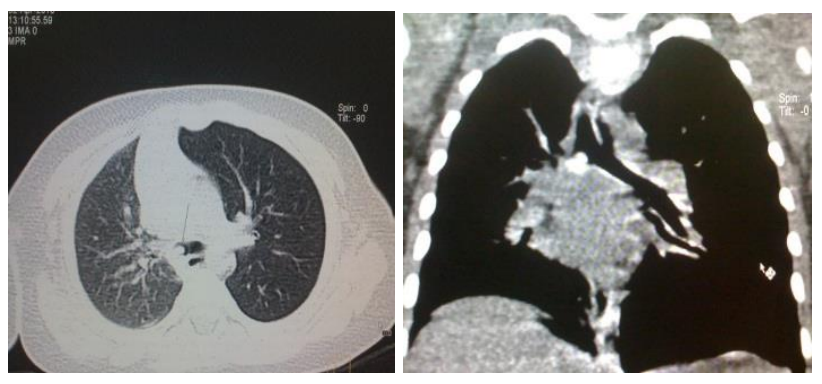

Figure 8: FB in right main bronchus with segmental collapse of lung.

Case 3

A nine months old child was brought to emergency department with history of accidental inhalation of a chickpea which the elder sibling (a four year old girl) gave to the infant. Infant initially had violent prolonged episode of cough which later on subsided after sometime. On examination the chest movements on right side were reduced along with decreased breath sounds. However, child was not in respiratory distress. To confirm the chest $\mathrm{X}$-Ray findings suggestive of a vegetative FB CT scan chest was done. It revealed vegetative FB in the right main bronchus causing segmental collapse of the lungs distal to foreign body (Figure 8). A three-dimensional reconstructed virtual bronchoscopy was also done to exactly localize the foreign body and it confirmed the findings of CT (Figure 9). The foreign body was removed by rigid bronchoscopy with the aid of foreign body grasping optical forceps as described in above two cases. 


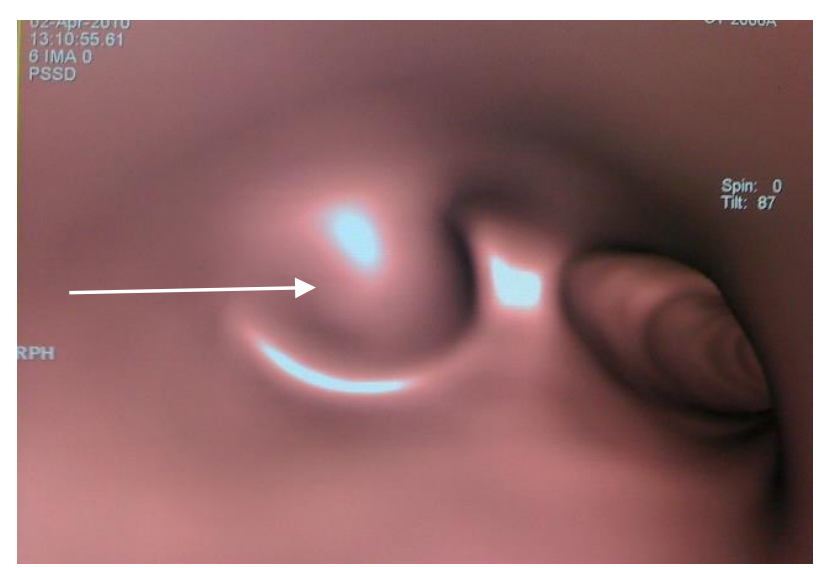

Figure 9: Virtual bronchoscopy localizing FB just beyond carina in the $\mathrm{Rt}$ main bronchus.

\section{DISCUSSION}

Foreign-body aspiration though not uncommon, can present as a life threatening emergency that warrants early removal. In 1897, Gustav Killian, a German otolaryngologist, performed the first bronchoscopy using a rigid esophagoscope to successfully remove a pig bone from a farmer's right main bronchus. ${ }^{2}$ Later, Chevalier Jackson developed bronchoscope with proximal illumination along with several specialized instruments for the removal of foreign bodies. ${ }^{2}$

Quite often, the diagnosis may be delayed, when the history is not forthcoming as parents have not witnessed FB inhalation or the choking episode is short-lived and acute symptoms subsides after a while, or parents simply forget to mention it. Sometimes clinician may be misguided when clinical and radiological findings are noncontributory or overlooked.

The delayed symptoms of FB in the aerodigestive tract may mimic other common conditions like asthma or recurrent pneumonia, therefore a high index of suspicion is crucial for an early diagnosis of foreign body aspiration in paediatric age group.

Younger children of age group one to three are at highest risk of FB aspiration. Boys are more likely to experience aspiration than girls: the male-to-female ratio varies from 1.5:1 to $2.4: 1$. $^{1}$ This has been attributed to several factors such as children tends to explore things by putting small objects into their mouths; they are often running, shouting, playing, with objects in their mouths; and lack of molars to chew adequately increases the risk of FB slipping into airway. ${ }^{3}$ Aspirated FB could be organic such as peanuts, seeds, vegetables or inorganic such as toy parts, crayons, metal screws etc. the list being inexhaustible.

Studies have shown that the right bronchus is more commonly affected than left as right bronchus is makes lesser angle of divergence and bigger diameter relative to the left bronchus. ${ }^{1}$

Signs and symptoms due to FB aspiration can be divided into 3 phases. ${ }^{1}$ Phase 1: Initial choking, coughing, gasping, and respiratory distress lasting for few seconds to minutes because of airway obstruction. Phase 2: Temporary quiescent phase in which, patient may not have any symptoms. These early symptoms resolve when the mucosal cough receptors accommodate. ${ }^{4}$ Phase 3: Symptoms of complications such as when infection sets in.

The radiology plays a vital role in aiding the diagnosis but when a positive history is lacking the role of radiology is unparalleled. Most of the foreign bodies aspirated are food materials and are radiolucent, therefore, indirect radiological findings should be carefully looked for. Chest $\mathrm{x}$-ray is taken during expiration as this makes the differences between lungs more prominent. Depending on degree of obstruction, a plain radiograph may show over inflation or an area of atelectasis. ${ }^{5}$ This happens due to check valve mechanism, where air enters the bronchus around the foreign body, gets trapped and is not expired out, leading to hyperinflation of the affected lung. Other findings could be rib flaring, mediastinal shift, lobar collapse or consolidation, unilateral emphysema or atelectasis; demonstrating a radio-opaque FB is uncommon. In our case series all the patients had the hyperinflation of lung on ipsilateral side along with mediastinal shift to opposite side.

In certain complicated cases due to missed or retained FB, multidetector computed tomogram (MDCT) can be done. Multiplanar reformatted images can delineate the exact shape, location and form of a bronchial FB which can help the surgeon in operative bronchoscopy and safe removal of FB. ${ }^{6}$ Occasionally, virtual bronchoscopy may be required. The images created are close to the real anatomy and help to identify and exactly localize the FB. If it is negative, it may reduce the number of unnecessary rigid bronchoscopies. ${ }^{7}$ However, bronchoscopy may still be required to be done in children with symptoms of recurrent LRTI even if radiology is non contributory. ${ }^{8}$ In a retrospective review of nearly 1900 bronchoscopies, $15 \%$ of the patients with a confirmed FB on bronchoscopy did not have a positive history. ${ }^{9}$

FB aspiration is one of the most challenging situations for both the anaesthesiologist and otoaryngologist, therefore it is imperative that both should discuss the procedural plan in detail. Rigid bronchoscopy is advantageous as it provides unrestricted visualization, good ventilatory control and ability to pass various instruments to remove the FB. A fasting of 6 hours for solids and 2 hours for clear fluids is preferred to decrease risk of aspiration during procedure. However, if the intervention has to be done urgently than, a large-bore gastric tube can be used 
to aspirate the stomach contents before induction of anaesthesia. ${ }^{10}$

Patient should be premedicated with an antisialagogue, injection glycopyrrolate (4 $\mu \mathrm{g} / \mathrm{kg}$ IV) to prevent secretions and injection dexamethasone $(0.4-1 \mathrm{mg} / \mathrm{kg} \mathrm{IV})$ to treat airway edema and inflammation after the procedure. The three main anaesthetic issues involve the methods of induction, ventilation during bronchoscopy, and maintenance of anesthesia. Induction technique may include inhalational or intravenous induction techniques. Controlled ventilation with muscle relaxant is a preferred technique for the removal of FB which are lodged distal of carina as the procedure will take a longer time. Spontaneous ventilation with topical anesthesia can be used for more proximal FB. ${ }^{10}$ Positive pressure ventilation in proximally lodged $\mathrm{FB}$ is not preferred by many as this increases risk of pushing the $\mathrm{FB}$ further distally into the airway. ${ }^{3}$ Maintenance of anaesthesia is achieved with oxygen, air with inhalational agent (sevoflurane, halothane).

Post retrieval of the FB endotracheal intubation is done to ventilate and re-expand any atelectasis of lungs and for pulmonary toileting. However, if the procedure has been short and uneventful then, simple mask ventilation with gentle chest physiotherapy in lateral position will prevent adverse airway event.

Within 6 to 8 hour post procedure, a check x-ray chest should be done to assess lung expansion, exclude pneumothorax and any residual foreign body. ${ }^{11}$

A potentially life threatening condition arises if FB being retrieved accidentally slips and completely blocks the tracheal lumen. The surgeon should maintain his composure and instead of struggling to get it out, a better idea would be to push the FB back to one of the bronchi and ventilate the other lung. The procedure can be reattempted once child is adequately ventilated. ${ }^{12}$ Following the removal of the foreign body, a check bronchoscopy is important to look for fragments or second foreign body and also to look for any bleeding caused due to instrumentation.

Thoracotomy is required when the FB cannot be removed by bronchoscopy, especially with more peripherally located FBs and when prior interventions have failed. The rate of thoracotomy as per one study was $2.1 \% .^{13}$

Fiber-optic bronchoscopy is traditionally avoided in paeditric age group, especially infants, as it blocks the airway lumen and it is not possible to ventilate the patient. It also has a limitation with respect to its small channel for instrumentation and suctioning. However, it is less invasive and is used in paediatric patients as a diagnostic tool, when the history, clinical and radiological examination is not contributory. ${ }^{14}$
Complications of retained airway FB depend on duration of elapsed time from aspiration to diagnosis. It includes, persistant wheeze, bronchiectasis, airway granulomas, bronchoesophageal fistula, and chronic aspiration leading to stricture. ${ }^{15}$

\section{CONCLUSION}

FB aspiration is one of the most common life threatening emergencies in paediatric age group. Targeted questions, a high degree of suspicion and prompt action is required to prevent long term complications. Our case series highlights the importance of meticulous history taking. The importance of closed loop functioning between the anaesthesiologist and otolaryngologist cannot be overemphasized.

\section{Funding: No funding sources \\ Conflict of interest: None declared \\ Ethical approval: Not required}

\section{REFERENCES}

1. Seth D, Kamat DM. Foreign-body aspiration: A guide to early detection, optimal therapy. Allergy. 2007;2:6.

2. Ramírez-Figueroa JL, Gochicoa-Rangel LG, Juan RS, David H, Vargas MH. Foreign body removal by flexible fiberoptic bronchoscopy in infants and children. Pediatric pulmonol. 2005;40(5):392-7.

3. Rovin JD, Rodgers BM. Pediatric foreign body aspiration. Pediatrics in review. 2000;21(3):86-90.

4. Louie MC, Bradin S. Foreign body ingestion and aspiration. Pediatr Rev. 2009;30:295-301.

5. Pinto A, Scaglione M, Pinto F, Guidi G, Pepe M, Del Prato B, et al. Tracheobronchial aspiration of foreign bodies: current indications for emergency plain chest radiography. La radiologia medica. 2006;111(4):497-506.

6. Bai W, Zhou X, Gao X, Shao C, Califano JA, Ha PK. Value of chest $\mathrm{CT}$ in the diagnosis and management of tracheobronchial foreign bodies. Pediatrics International. 2011;53(4):515-8.

7. Haliloglu M, Ciftci AO, Oto A, Gumus B, Tanyel FC, Senocak ME, et al. CT virtual bronchoscopy in the evaluation of children with suspected foreign body aspiration. European journal of radiology. 2003;48(2):188-92.

8. Cohen S, Avital A, Godfrey S, Gross M, Kerem E, Springer C. Suspected foreign body inhalation in children: what are the indications for bronchoscopy? J pediat. 2009;155(2):276-80.

9. Aydoğan LB, Tuncer U, Soylu L, Kiroğlu M, Ozsahinoglu C. Rigid bronchoscopy for the suspicion of foreign body in the airway. Int $\mathrm{J}$ Pediatric Otorhinolaryngol. 2006;70(5):823-8.

10. Fidkowski CW, Zheng H, Firth PG. The anesthetic considerations of tracheobronchial foreign bodies in children: a literature review of 12,979 cases. Anesthes Analg. 2010;111(4):1016-25. 
11. Kalyanappagol VT, Kulkarni NH, Bidri LH. Management of tracheobronchial foreign body aspirations in paediatric age group-A 10 year retrospective analysis. Indian $\mathrm{J}$ Anaesthes. 2007;51(1):20.

12. Gang W, Zhengxia P, Hongbo L, Yonggang L, Jiangtao D, Shengde W, et al. Diagnosis and treatment of tracheobronchial foreign bodies in 1024 children. J Pediat Surg. 2012;47(11):2004-10.

13. Soysal O, Kuzucu A, Ulutas H. Tracheobronchial foreign body aspiration: a continuing challenge. Otolaryngol Head Neck Surg. 2006;135(2):223-6.

14. Wood RE, Gauderer MW. Flexible fiberoptic bronchoscopy in the management of tracheobronchial foreign bodies in children: the value of a combined approach with open tube bronchoscopy. J Pediatr Surg. 1984;19(6):693-8.

15. Reilly J, Thompson J, Macarthur C, Pransky S, Beste D, Smith M, et al. Pediatric aerodigestive foreign body injuries are complications related to timeliness of diagnosis. Laryngoscope. 1997;107(1):17-20.

Cite this article as: Dwivedi G, Gupta V, Dwivedi D, Sood A, Upadhyay M, Kumar M. Retrieval of foreign body bronchus in paediatric age group: a challenge. Int J Otorhinolaryngol Head Neck Surg 2018;4:1115-20. 